\title{
Charged dust and shock phenomena in the Solar System
}

\author{
S. I. Popel ${ }^{1}$ and A. A. Gisko ${ }^{2}$ \\ ${ }^{1}$ Institute for Dynamics of Geospheres, Russian Academy of Sciences, Moscow, Russia \\ ${ }^{2}$ Prokhorov Institute of General Physics, Russian Academy of Sciences, Moscow, Russia \\ Received: 16 January 2006 - Revised: 3 April 2006 - Accepted: 4 April 2006 - Published: 21 June 2006
}

\begin{abstract}
The results on shock phenomena in dusty plasmas of the Solar System are reviewed. The problems of dust ion acoustic bow shock in interaction of the solar wind with dusty cometary coma and formation of transient atmospheres of atmosphereless cosmic bodies such as Moon, Mercury, asteroids and comets are considered. The latter assumes the evolution of meteoroid impact plumes and production of charged dust grains due to the condensation of both the plume substance and the vapor thrown from the crater and the surrounding regolith layer. Physical phenomena occurring during large meteoroid impacts can be modeled with the aid of active rocket experiments, which involve the release of some gaseous substance in near-Earth space. New vistas in investigation of shock processes in natural dusty plasmas are determined.
\end{abstract}

\section{Introduction}

Dusty plasmas represent the most general type of plasmas in our Solar System. In fact, it is difficult to find any plasma environment in the Solar System that is free of dust particles - perhaps the interior of the Sun is the only exception. The brightness observations of the F-corona, made during total solar eclipses or from satellites equipped with coronagraphs have shown the possibility of the innermost dust cloud at the distances from the Sun not exceeding $0.3 \mathrm{AU}$ (Kimura, 1997; Mann, 2000). The sources of the dust are asteroids, short-period comets as well as the interplanetary dust which is present at larger distances from the Sun than the innermost dust cloud. The probable origin of the interplanetary dust is from decay by collisional fragmentation of debris from comets, micrometeoroids, man-made pollution, etc. The main composition of the interplanetary and cometary dust is $60 \%$ chondritic $(\mathrm{Fe}, \mathrm{Mg}, \mathrm{Si}, \mathrm{C}, \mathrm{S}), 30 \%$

Correspondence to: S. I. Popel

(s_i_popel@mtu-net.ru) iron-sulphur-nickel, and $10 \%$ silicates (olivine). The parameters of the interplanetary dust are the size $a \sim 1-10 \mu \mathrm{m}$, the density $n \sim 10^{-10}-10^{-9} \mathrm{~cm}^{-3}$. The dust environment is denser in the vicinity of planets, and dust is most prominently seen in the ring systems surrounding all giant planets of our Solar System (Krüger, 2003). In the dust evolution cycle, dust offers a tangible, physical link between our planetary system and the stars. For example, dust is formed in the extended envelopes of evolved stars, and it is an intimate player in proto-planetary accretion discs and the formation of planetesimals. Therefore, the study of dust in space can provide important information on the fundamental processes governing the formation of planetary systems.

The dust grains in space acquire variable charges due to the competing effects of electron and ion capture from the ambient plasma, secondary electron emission from the impacts of high energetic ions or electrons, on the release of photoelectrons from the ambient UV radiation field (Ostrikov et al., 1999, 2000; Verheest, 2000). The dust particle charging process allows us to consider most plasmas in the Solar System containing dust as dusty plasmas consisting of electrons, ions, neutrals, and charged dust grains. This process is a basis for the strong dissipativity of the dusty plasma system (Tsytovich, 1997). The strong dissipativity results in a new physics of nonlinear wave structures in dusty plasmas. First, anomalous dissipation originating from dust grain charging appears. Second, new kinds of nonlinearities take place. The anomalous dissipation leads to preferential importance of shock waves in dusty plasmas, which have specific features that distinguish them from ordinary collisional and collisionless shock waves. That dust ion acoustic shock waves associated with anomalous dissipation can actually exist was proved analytically by Popel et al. (1996, 2001, 2005) and experimentally by Nakamura et al. (1999) and Luo et al. (1999).

At present, at least two natural phenomena which illustrate the importance of shocks, where dust charging effect is important, are known. These are dust ion acoustic

Published by Copernicus GmbH on behalf of the European Geosciences Union and the American Geophysical Union. 
bow shock formation in interaction of the solar wind with dusty cometary coma (Popel et al., 2003) and shock wave structures associated with the evolution of meteoroid impact plumes and production of charged dust grains due to the condensation of both the plume substance and the vapor thrown from the crater and the surrounding regolith layer in the problem of formation of transient atmospheres of atmosphereless cosmic bodies such as Moon, Mercury, asteroids and comets (Nemtchinov et al., 2002). In Sects. 2 and 3 of this paper we review briefly these possibilities. We bring also the reader's mind to bear on the fact that physical phenomena occurring during a large meteoroid impact with the Moon's surface can be modeled with the aid of active rocket experiments, which involve the release of some gaseous substance in near-Earth space, and present some data on such experiments (Sect. 4). Furthermore, in Sect. 5 we discuss possible phenomena in nature related to the expanding dusty plasmas where the processes of dust grain production and shocks are important.

\section{Dust ion acoustic bow shock}

Theoretical studies of the interaction of the solar wind with cometary comae do not take into account usually the influence of dust. Cometary nuclei are found to be, most likely, dominated by refractories, very porous and fragile (though monolitic rather than of rubble-pile structure), and generally quite homogeneous except for dust mantling and the effects of radial migration of volatiles in the interiors (Rickman, 1998). Under the action of the solar light volatile components of the cometary nucleus evaporate and vapor stream entrains dust particles.

The dust/gas ratio is introduced to characterize the ratio of masses of refractories to volatiles in the cometary nuclei. In quantitative terms, an average value of dust/gas ratio exceeds unity (Kührt and Keller, 1996). The dust/gas ratio obtained from the coma observations is in the range 0.1-1 (Newburn Jr. and Spinard, 1989; Singh et al., 1992; Storrs et al., 1992). Thus we can expect significant quantity of dust in the cometary coma (tens per cent of the total mass of the coma). Assuming ten per cent content of the dust in the cometary substance and bulk density of about $1 \mathrm{~g} / \mathrm{cm}^{3}$, we obtain that the density of micron-sized particles is of the order of $10^{11} \mathrm{~cm}^{-3}$ (Losseva et al., 2002).

The crucial point of the investigation of the interaction of the solar wind with cometary comae is the description of bow shock formed as a result of this interaction. The presence of dust constituting tens per cent of the total mass of the coma can modify the bow shock. Indeed, it is well known that in laboratory dusty plasmas dust often contributes only a few percent of the mass density. The investigations in laboratory dusty plasmas (Popel et al., 1996; Nakamura et al., 1999; Luo et al., 1999) show that even for small mass density of the dust, it can be the crucial factor in shock formation and propagation. Thus the problem of the investigation of the in- fluence of the dust on the bow shock follows from the problem of the investigation of laboratory dusty plasmas.

The interaction of the solar wind with comets is important only when the comet has rather extended and dense atmosphere. The atmosphere expands in the ambient gas of a very low pressure. The main reason for an appearance of the atmosphere is the evaporation of solid (volatile) substance constituting the cometary nucleus under the action of the solar radiation. The refractories from the destructing cometary nucleus enter also the atmosphere and represent its dust fraction. The density of volatiles $\left(\mathrm{H}_{2} \mathrm{O}, \mathrm{CO}_{2}\right.$, $\mathrm{CH}_{4}$, etc.) is determined as a rule theoretically (Houpis and Mendis, 1981). The size distribution of coma grains is such that the total area is dominated by the smallest grains present, and the 1P/Halley in-situ results showed that this continued to hold down to radii $a \sim 0.01 \mu \mathrm{m}$ (Mazets et al., 1987; Vaisberg et al., 1987). The size distribution is well determined observationally (Grün and Jessberger, 1990) as an approximate power-law function with index $\sim-4$ in terms of radius. We consider in all subsequent estimates and calculations the characteristic dust grain size as $a \sim 1 \mu \mathrm{m}$. Due to the processes of photoionization the gas surrounding the comet becomes ionized at the distances exceeding several kilometers from the cometary nucleus (Houpis and Mendis, 1981). It is the interaction between the cometary ions (the dominant are the ions $\mathrm{H}_{3} \mathrm{O}^{+}$) and the solar wind protons that is considered as the strongest one in the interaction between the cometary coma and the solar wind. This is related to the fact that the collision frequency between the ions of the coma and the solar wind is higher than the frequencies between the charged and the neutral particles. Because the main particles participating in the interaction are ions, one may conclude that the bow shock is the ion acoustic bow shock. The presence of dust in the coma leads to another important kind of interaction - the interaction between the solar wind protons and charged dust of the coma. The dust particle charging process is described by the equation

$\partial_{t} q_{d}=I_{e}\left(q_{d}\right)+I_{i}^{S W}\left(q_{d}\right)+I_{i}^{c}\left(q_{d}\right)+I_{p h}\left(q_{d}\right)$,

where $q_{d}=Z_{d} e$ is the dust particle charge; $I_{e}, I_{i}^{S W}, I_{i}^{c}$ are the microscopic grain currents (originating from the potential difference between the plasma and the grain surface) of electrons, solar wind ions, and cometary coma ions, respectively; $I_{p h}$ is the photoelectric current of electrons emitted from the dust grain surface due to the presence of external electromagnetic radiation: for their explicit expressions see, e.g., (Popel et al., 2000); $-e$ is the electron charge.

The ions of the coma together with the charged dust interact hydrodynamically with the ions of the solar wind. This allows us to determine the distance $R$ from the cometary nucleus where the bow shock appears. For the parameters of the solar radiation spectrum in the vicinity of the Earth, electron and ion temperature in the solar wind $T_{e}^{S W}=T_{i}^{S W}=15 \mathrm{eV}$, the ion temperature in the cometary coma $T_{i}^{c}=0.03 \mathrm{eV}$, the electron and ion (proton) density in 


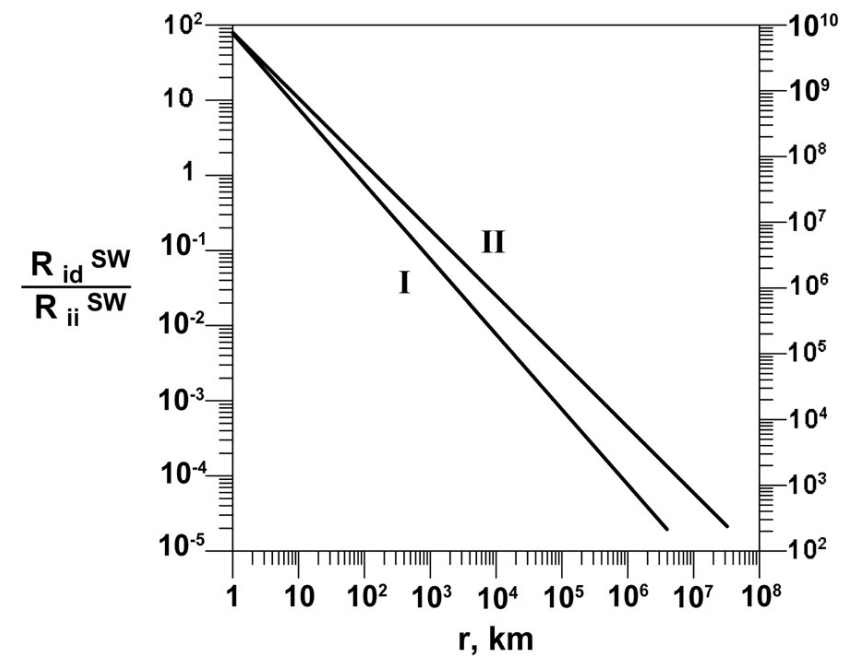

Fig. 1. The ratio $R_{i d}^{S W} / R_{i i}^{S W}$ as a function of the distance from the cometary nucleus. The lines I and II correspond to the dust densities near the nucleus $10^{3} \mathrm{~cm}^{-3}$ and $10^{11} \mathrm{~cm}^{-3}$, respectively (the ratio magnitudes for the lines I and II are shown on the left and right vertical axes, respectively).

the solar wind $n_{e}^{S W} \sim n_{p}=20 \mathrm{~cm}^{-3}$, the ion density in the coma near the nucleus $n_{i 0}^{c}=1.43 \times 10^{5} \mathrm{~cm}^{-3}$, the ion density in the coma depending on the distance $r$ from the nucleus as $n_{i}^{c}=n_{i 0}^{c} \times\left(r_{0} / r\right)$, the radius of the nucleus $r_{0}=1 \mathrm{~km}$, and $\mathrm{H}_{3} \mathrm{O}^{+}$cometary coma ions, we find that $R \sim 10^{4} \mathrm{~km}$ and the dust particles acquire positive charges reaching the values exceeding $Z_{d} e=2000 e$ for $r \approx R$. The equation describing the momentum transfer of the solar wind protons takes the form

$m_{p} n_{p} \frac{d V_{p \alpha}}{d t}=-\frac{\partial p_{p}}{\partial x_{\alpha}}+\eta^{S W} \frac{\partial W_{p \alpha \beta}}{\partial x_{\beta}}-e n_{p} \frac{\partial \varphi}{\partial x_{\alpha}}+R_{i d \alpha}^{S W}+R_{i i \alpha}^{S W}$,

where the Greek letters denote vector (tensor) component,

$W_{p \alpha \beta}=\frac{\partial V_{p \alpha}}{\partial x_{\beta}}+\frac{\partial V_{p \beta}}{\partial x_{\alpha}}-\frac{2}{3} \delta_{\alpha \beta} \nabla \cdot V$,

$m_{p}$ is the proton mass, $\mathbf{V}$ is the ion speed, $\phi$ is the electrostatic potential, $p_{p}$ is the proton pressure, $\eta^{S W}$ and $\mathbf{R}_{i i}^{S W} \approx-m_{p} n_{p} v\left(\mathbf{V}_{p}\right)\left(\mathbf{V}_{p}-\mathbf{V}_{c}\right)$ are respectively the viscosity and the friction force related to the interaction between the solar wind protons and cometary coma ions, $\mathbf{R}_{i d}^{S W} \approx-$ $m_{p} I_{i}^{S W} n_{d}\left(\mathbf{V}_{p}-\mathbf{V}_{d}\right) / e$ is the friction force related to the interaction between the solar wind protons and cometary coma dust, $v\left(\mathbf{V}_{p}\right) \approx 4 \pi e^{4} n_{i}^{c} L / m_{p}^{2} V_{p}^{3}$ is the Coulomb collision frequency between the solar wind protons and the cometary coma ions, $L$ is the Coulomb logarithm, $\mathbf{V}_{c(d)}$ is the speed of the cometary coma ions (dust grains), $n_{d} \propto\left(r_{0} / r\right)^{2}$ is the dust density. The terms containing $\eta^{S W}$ and $\mathbf{R}_{i i}^{S W}$ on the right-hand side of Eq. (2) are of the same order of magnitude. Thus if $R_{i d}^{S W} \equiv\left|\mathbf{R}_{i d}^{S W}\right|$ exceeds $R_{i i}^{S W} \equiv\left|\mathbf{R}_{i i}^{S W}\right|$ then the interacion of dust with the solar wind protons dominates. In Fig. 1 the ratio $R_{i d}^{S W} / R_{i i}^{S W}$ is presented for different distances

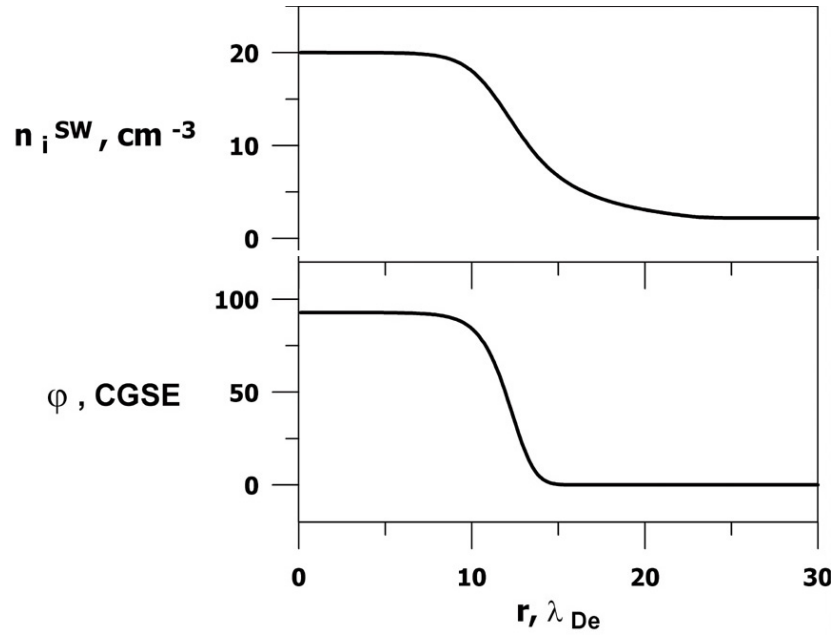

Fig. 2. Profiles of the solar wind ion density $n_{i}$ and the electrostatic potential $\phi$ in a bow shock structure. The spatial variable $r$ is expressed in units of the electron Debye length $\lambda$ De.

from the cometary nucleus for the above plasma parameters. The effects of dust-proton interaction become dominant in the region of bow shock for the dust densities exceeding $10^{6} \mathrm{~cm}^{-3}$. The structure of the bow shock is described by the set of equations where, in addition to Eqs. (1) and (2), the continuity equation for the solar wind protons is included, the cometary coma ions are described by the continuity and momentum transfer equations, the electron density is taken to be Boltzmann with constant $T_{e}$, and the Poisson's equation is used. This model is a generalization of the model applied for the study of shock-like structures in charge-varying dusty plasmas in the presence of the electromagnetic radiation (Popel et al., 2000) but developed with taking into account the two sorts of ions. In Fig. 2 shock profiles are given which are obtained for $r \sim 10^{4} \mathrm{~km}$. We note that there is a good agreement between the estimate for the shock front width $\Delta \xi \sim c_{s} / v_{q}$, where $c_{s}=\sqrt{T_{e}^{S W} / m_{p}}$ is the ion acoustic speed in the solar wind, $v_{q}$ is the dust charging frequency

$v_{q} \equiv-\left[\partial\left(I_{e}\left(q_{d}\right)+I_{i}^{S W}\left(q_{d}\right)+I_{i}^{c}\left(q_{d}\right)+I_{p h}\left(q_{d}\right)\right) / \partial q_{d}\right]_{q_{d}=q_{e q}}$,

where $q_{e q}$ is the equilibrium dust particle charge. Thus for typical cometary nucleus size of about $1 \mathrm{~km}$ and rather dense dusty coma $\left(n_{d}>10^{6} \mathrm{~cm}^{-3}\right)$ the bow shock formed as a result of the interaction of the solar wind with the coma is expected to be related to the anomalous dissipation due to the dust particle charging. The bow shock is similar, by its origin, to the shocks observed by Luo et al. (1999) and Nakamura et al. (1999) and those predicted theoretically by Popel et al. $(1996,2000)$. 


\section{Large meteoroid impacts and transient atmospheres}

Another phenomenon in the Solar System related to shock formation with participation of charged dust is a production of transient atmospheres of atmosphereless cosmic bodies (Moon, Mercury, asteroid and comet) as a result of impacts of large meteoroids or man-made projectiles with these bodies. A local transient atmosphere has been discovered near the Moon surface (Potter Jr. and Morgan, 1988). Three types of a steady atmosphere sources have been proposed, namely, impact vaporisation by micrometeoroids, solar wind sputtering, and photodesorption (Smyth and Marconi, 1995; Mendillo et al., 1999).

Meteoroid impact is accompanied by emission of electromagnetic radiation which in the case of rather large meteoroids can be detected even by the Earth's observers. The impact can be responsible for the optical flashes on the Moon surface. Furthermore, the evolution of the impact generated vapor plume can lead to such effects as the expulsion of the interplanetary magnetic field, formation of the collisionless shock wave structure associated with intense plasma turbulence, electron heating, and even the production of x-rays. The impacts of large meteoroids can, in particular, occur during the meteor storms.

To describe vapor jet we use the results of simulations (Nemtchinov et al., 1998, 1999). We consider an impact of the meteoroid of the size of $1-10 \mathrm{~cm}$ with the Moon surface. For an impactor body of $10 \mathrm{~cm}$ size and its velocity $20 \mathrm{~km} / \mathrm{s}$ the impact generated plume has a conical form, at the moment of $2.5 \mathrm{~s}$ after the impact the dense core of the plume and the radius being about $10 \mathrm{~km}$ and $5 \mathrm{~km}$, respectively, while the density of the vapor core being $10^{-15} \mathrm{~g} / \mathrm{cm}^{3}$. After that moment the collisionless phase of the plume expansion starts. If the vapor plume expands in the region of the presence of the solar wind the latter begins to penetrate in the vapor plume and cause the dissociative ionization of the vapor molecules. The density of the molecules is $2 \times 10^{7} \mathrm{~cm}^{-3}$. This value is approximately six orders of magnitude higher than the corresponding one of the solar wind in the vicinity of the Moon. The values of the density in the vapor plume and in the solar wind become approximately equal to each other when the length of the plume reaches the value of 1000 $\mathrm{km}$ while the radius of $500 \mathrm{~km}$. This occurs at the moment of about $250 \mathrm{~s}$.

The evolution of the impact plume can lead to the formation of a shock wave structure associated with an appearance of charged grains (macroparticles). The shock wave front is associated with the fore (border)-part of the plume. The first type of the macroparticles (small droplets) is created as a result of the process of condensation which takes place during the expansion of the vapor plume. The period of the formation of the centers of condensation is very short and all droplets have approximately the same size $a \approx 3 \mu \mathrm{m}$. The degree of condensation is equal approximately to $0.2-$ 0.3 . The droplets move together with the substance of the plume. Their speed is about $3-5 \mathrm{~km} / \mathrm{s}$. This value exceeds the first astronautical velocity for the Moon, $2.3 \mathrm{~km} / \mathrm{s}$, and the droplets will not fall down the surface of the Moon. The second type of the macroparticles (dusts) is thrown from the crater and surrounding it regolith layer. Typical size of the lunar dust is about $30 \mu \mathrm{m}$, the velocity is within the range $0.3-1 \mathrm{~km} / \mathrm{s}$. The estimate of the mass ejected due to the impact gives the value which is 100-1000 times higher than the impactor mass. Assuming the lower number, or the mass of the matter ejected of about $100 \mathrm{~kg}$ we find the number of the grains $N \approx 4 \times 10^{11}$. The gravity restricts the rise of such grains. For the lunar gravity $160 \mathrm{~cm} / \mathrm{s}^{2}$ and the speed $0.3 \mathrm{~km} / \mathrm{s}$ the dust is stopped and falls back in approximately $20 \mathrm{~s}$. The maximum altitude attained by the grains is about $3 \mathrm{~km}$. One can expect the charging of the grains. This process is caused by the flows of electrons and protons of the solar wind on them. If the expansion of the vapor plume occurs on the sunlit side of the Moon then the contribution of the photoelectric effect is important and the macroparticles acquire positive charges. In this case the maximum charge is of the order of $10^{4}$ electron charge for the droplets and $10^{5}$ for the dusts. If one can neglect the photoelectric effect the macroparticles acquire negative charges, the maximum charge being of the order of $10^{5}$ electron charge for the droplets and $10^{6}$ for the dusts.

The detection of fast electron formation and x-ray flux at the head of the comet Hyakutake (Dawson et al., 1997) and plasma oscillations at the boundary of diamagnetic cavity of the artificial comet AMPTE caused by barium release (Bingham et al., 1991; Popel et al., 1995) are the basis for our hypothesis of the plasma turbulence in large regions of the solar wind above the lunar surface.

Once ionized, a molecule in the vapor plume finds itself in the solar wind flow. The instability similar to an ion-ion twostream instability occurs. The energy source of the instability is the relative motion of the vapor plume and the solar wind ions. The frequency of the waves excited is close to the lower hybrid frequency $\omega_{l h}$,

$\omega_{l h}^{2}=\omega_{p i}^{2} /\left(1+\omega_{p e}^{2} / \omega_{c e}^{2}\right) \approx \omega_{c e} \omega_{c i}$,

where $\omega_{p e(i)}$ is the electron (ion) plasma frequency, $\omega_{c e(i)}$ is the electron (ion) gyrofrequency. The situation is similar to those which occur in the process of the AMPTE barium release (Bingham et al., 1991; Popel et al., 1995) and interaction of the solar wind with the coma of the comet Hyakutake (Dawson et al., 1997).

The significant difference is the influence of the charged macroparticles on the process of the instability development. The waves are excited in the region near the front of the expanding vapor jet. The width of this turbulent region is estimated to be of the order of $10-100 \mathrm{~km}$. In the turbulent region which corresponds to the compression region of the collisionless shock wave (Bingham et al., 1991; Popel et al., 1995) one can expect the increase of the magnetic field while inside the vapor jet the magnetic field is decreased and the 
formation of the diamagnetic cavity is possible. The development of the plasma turbulence results in the electron heating. As a result, high-energy (with the energies up to several $\mathrm{keV}$ ) electrons appear. Part of these electrons moves toward the surface of the Moon and falls on it. The slowing down these electrons gives bremsstrahlung with some $\mathrm{x}$-ray photons. The power of $\mathrm{x}$-ray radiation is of order $1 \mathrm{~kW}$. The process of electromagnetic radiation lasts during dozens of seconds - several minutes. We note that because in the process of the electron heating not only the high-energy electrons appear but also the electrons with lower energies are generated one can expect the electromagnetic radiation also in optical and UV ranges.

We note that a $1-\mathrm{m}$ sized impactor hits the Moon about one or two times per year. For this case, the size of the perturbation zone would be about $1000 \mathrm{~km}$, comparable to the size of the Moon. The Moon is the first cosmic object not only studied but used by humanity for various purposes, e.g., for lunar bases. Debris caused by impacts and electrostatic charging and electromagnetic waves due to the plasma turbulence may be important hazardous factors, e.g., for radio communications.

In Fig. 3, the portions of the impact processes described above (optical photons appeared due to the impact, plume formation and evolution, macroparticles formation, zone of plasma turbulence in the region of the solar wind interaction with the plume, generation of fast electrons as well as the UV and x-ray photons) are presented schematically.

\section{Active rocket experiments in near-Earth space}

Physical phenomena occurring during a large meteoroid impact with the Moon's surface can be modeled with the aid of active rocket experiments, which involve the release of some gaseous substance in near-Earth space. The source for the substance release in the ionosphere in these experiments is the generator of high-speed plasma jets. Their scheme is analogous to that of the "Fluxus" and "North Star" experiments carried out at altitudes of 140,280 , and $360 \mathrm{~km}$ (Gavrilov et al., 1998; Erlandson et al., 1999).

The idea of application of such experiments to the problem of formation of shocks related to dust charging was forwarded in the papers (Popel and Tsytovich, 1999; Popel et al., 2000). The optimum altitudes for the experiments allowing us to observe the dust ion acoustic shocks are 500 $600 \mathrm{~km}$. The shock wave front is associated with the fore (border)-part of the jet propagating in the plasma of the ionosphere. The optimum speeds of the jet are $U \sim 10 \mathrm{~km} / \mathrm{s}$. Macroparticles (drops) appear as a result of condensation. The formation of the macroparticles in the active rocket experiments is confirmed by the results of both active and (modeling them) laboratory experiments as well as numerical simulations. In the first injection of the "North Star" experiment positively charged clusters of aluminium consisting

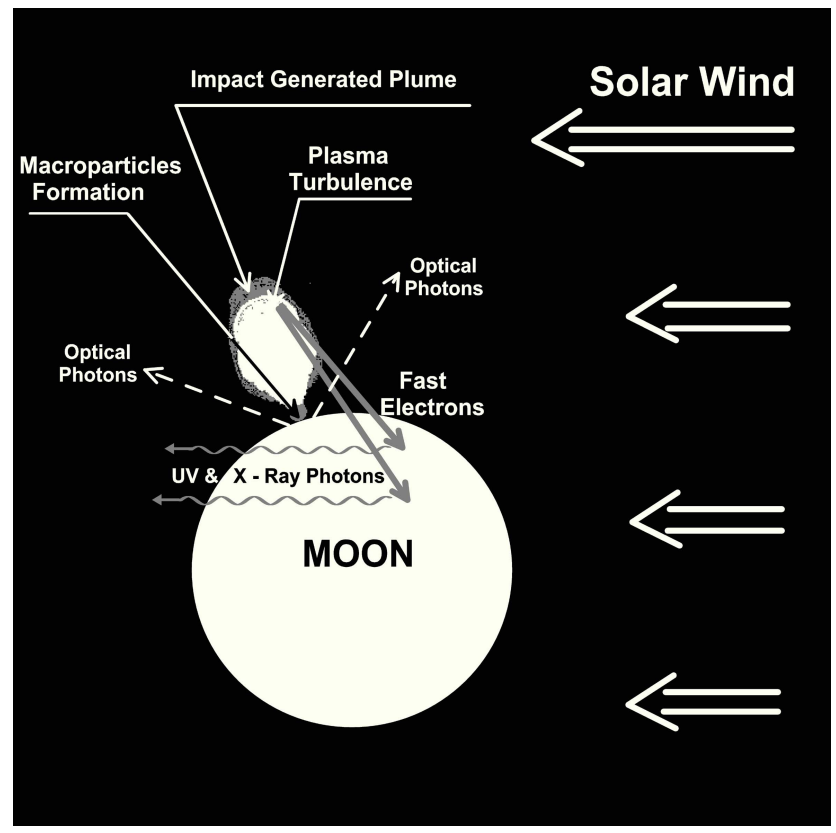

Fig. 3. The portions of the impact processes: optical photons appeared due to the meteoroid impact onto the Moon, plume formation and evolution, macroparticles formation in the plume, zone of plasma turbulence in the region of the solar wind interaction with the plume, generation of fast electrons as well as the UV and x-ray photons.

of $2,4,8$, and 16 atoms were detected in the jet (Kiselev et al., 2006). The clusters of larger masses could not be detected because of the narrow range of the measured cluster energies. However the analysis (Zetzer et al., 2006) of electromagnetic radiation from the jet in the infra-red range of the spectrum shows the presence of micrometer-sized charged drops. The drops are charged due to their interaction with the ambient plasma and the photoelectric effect.

\section{Summary and new vistas}

The examples we have given make clear the nature of shock phenomena in dusty plasmas of our Solar System. We have presented the results of the investigations of the dust ion acoustic bow shock formed in interaction of the solar wind with dusty cometary coma. Furthermore, we have considered expanding dusty plasmas with respect to the problem of transient atmosphere formation and the active geophysical rocket experiments, which involve the release of some gaseous substance in near-Earth space.

New vistas in investigation of natural dusty plasmas are related to further investigations of expanding dusty plasmas. An expanding dusty plasma formed in strong perturbations is even a more complicated system than the usual dusty plasmas because the evolution of the expanding dusty plasma includes self-consistently the process of dust grain production. 
However, the study of the expanding dusty plasmas formed in strong perturbations is very important that is dictated by the applications related to the description of different natural phenomena. Here we outline two problems which can be important for applications of expanding dusty plasmas in nature:

\section{Shocks in supernova explosions.}

The layer of dust behind the supernova shock is often observed. The problem is to verify whether the layer of dust is related to the process of dust condensation behind the shock wave front.

2. Nano- and microscale dust particles in the origin of the primary Earth's crust, hydrosphere, and atmosphere.

Theory and the data of comparative planetology as well as the Earth sciences (consistent with each other) enable us to state that the formation of the terrestrial planets occurred during 30-100 million years beginning from an appearance of the first condensate approximately $4.6 \times 10^{9}$ years ago (Vitjazev et al., 1990). During the Earth growth the main energy source was related to impacts of cosmic bodies falling to the growing planet. The mass spectrum of the cosmic bodies is considered to be proportional to $m^{-q}$ where $q \approx 1.8 \pm 0.2$. The main mass is concentrated in the bodies with diameters of $100 \mathrm{~km}$ and larger. Craters formed as a result of the impact of such a large bodies with Earth's surface are estimated to have depths of the order of the Earth's diameter. The matter of the impact generated vapor plume has the mass of the order of the cosmic body (impacting the Earth) while its temperature reaches several electronVolts. All this matter expands (lifts) with subsequent ionization, cooling, condensation, formation of nanoand microscale dust particles, and their charging. Thus the dusty plasma is formed. The important problem is to determine the characteristic sizes of the dust particles and estimate their mass spectrum because these will allow us to determine behavior of the dust grains: whether they work to the primary Earth's surface or they evolve in the primary atmosphere. This study will allow us to update the scenario of the formation of the primary Earth's crust, hydrosphere, and atmosphere and possibly to modify the scenario of the origin of terrestrial planets.

Furthermore, some laboratory studies of dusty plasmas could be important from the viewpoint of the problems discussed in this paper:

1. We have noted the importance of the laboratory experiments (Nakamura et al., 1999; Luo et al., 1999) on the dust ion acoustic shocks from the viewpoint of the interaction of the solar wind with dusty cometary coma. In these experiments the dust was always negatively charged. In the application to the dust ion acoustic bow shock the dust particles acquire a positive charge due to the photoelectric effect. Thus laboratory experiments on the dust ion acoustic linear and nonlinear waves (including shocks) in a dusty plasma with positive charges of the dust particles would be important.

2. The presence of charged nanoscale particles in a dusty plasma modifies its properties (Vladimirov and Ostrikov, 2004; Ostrikov, 2005). Thus further laboratory investigations of dusty plasmas containing nanoscale particles would be useful from the viewpoint of the analysis of the processes of the formation of the primary Earth's crust, hydrosphere, and atmosphere.

Acknowledgements. This work was carried out within the Program of Fundamental Investigations of the Division of Earth Sciences of the Russian Academy of Sciences "Nanoparticles in Natural and Technogenic Systems" and was supported by the Russian Foundation for Basic Research, project no. 06-05-64826-a. One of the authors (S. I. Popel) would like to thank the Humboldt Foundation for the equipment donation (Gerätespende V-8151/02093). The equipment (FFT CCD-Camera) was used in laboratory experiments modeling the active geophysical those. S. I. Popel is also grateful to the Russian Science Support Foundation, grant in the nomination "Doctors of Science of the Russian Academy of Sciences". The authors would like to thank T. V. Losseva and S. V. Vladimirov for fruitful discussions.

Edited by: N. S. Erokhin

Reviewed by: three referees

\section{References}

Bingham, R., Shapiro, V. D., Tsytovich, V. N., de Angelis, U., Gilman, M., and Shevchenko, V. I.: Theory of wave activity occurring in the AMPTE artificial comet, Phys. Fluids, 3, 17281738, 1991.

Dawson, J. M., Bingham, R., and Shapiro, V. D.: X-rays from comet Hyakutake, Plasma Phys. Controlled Fusion, 39, A185193, 1997.

Erlandson, R. E., Swaminathan, P. K., Meng, C.-I., Stoyanov, B. J., Zetzer, J. I., Gavrilov, B. G., Kiselev, Yu. N., and Romanovsky, Yu. A.: Observation of Auroral emissions induced by artificial plasma jets, Geophys. Res. Lett., 26, 1553-1556, 1999.

Gavrilov, B. G., Erlandson, R. E., Kiselev, Y. N., Meng, C.-I., Podgorny, I. M., Sobyanin, D. B., and Zetzer, J. I.: Dynamics of high energy plasma jet in the space: In situ experiment and laboratory simulation, Adv. Space Res., 21, 773-776, 1998.

Grün, E. and Jessberger, E.: Dust, in: Physics and Chemistry of Comets, Springer, Berlin, 113-176, 1990.

Houpis, H. L. F. and Mendis, D. A.: On the development and global oscillations of cometary ionospheres, Astrophys. J., 243, 1088$1102,1981$.

Kimura, H., Ishimoto, H., and Mukai, T.: A study on solar dust ring formation based on fractal dust models, Astron. Astrophys., 326, 263-270, 1997.

Kiselev, Y. N., Kosarev, I. B., and Poklad, Y. V.: Formation of nanoclusters and their interaction with environment in active geo- 
physical experiments, in: Nano- and Microscale particles in Geophysical Processes, edited by: Adushkin, V. V. and Popel, S. I., The Moscow Inst. of Physics and Technology (in Russian), Moscow, 123-133, 2006.

Krüger, H.: Jupiter's Dust Disc. An Astrophysical Laboratory, Shaker Verlag, Aachen, 2003.

Kührt, E. K. and Keller, H. U.: On the importance of dust in cometary nuclei, in: Worlds in Interaction: Small Bodies and Planets of the Solar System, Kluwer, Dordrecht, 79-89, 1996.

Losseva, T. V., Golub', A. P., Kosarev, I. B., Popel, S. I., and Nemtchinov, I. V.: Physical and Gasdynamical Processes Caused by Cometary Impacts onto the Sun, in: Proceedings of the Conference Asteroids, Comets, Meteors - ACM2002, European Space Agency, 873-876, 2002.

Luo, Q.-Z., D'Angelo, N., and Merlino, R. L.: Experimental study of shock formation in a dusty plasma, Phys. Plasmas, 6, 3455$3458,1999$.

Mann, I., Krivov, A., and Kimura, H.: Dust cloud near the Sun, Icarus, 146, 568-582, 2000.

Mazets, E. P., Sagdeev, R. Z., Aptekar, R. L., Golenetskii, S. V., Guryan, Y. A., Dyatchkov, A. V. Ilyinskii, V. N., Panov, V. N., Petrov, G. G., Savvin, A. V., Sokolov, I. A., Frederiks, D. D., Khavenson, N. G., Shapiro, V. D., and Shevchenko, V. I.: Dust in comet P/Halley from Vega observations, Astron. Astrophys., 187, 699-706, 1987.

Mendillo, M., Baumgardner, J., and Wilson, J.: Observational test for the solar wind sputtering origin on the Moon's extended sodium atmosphere, Icarus, 137, 13-23, 1999.

Nakamura, Y., Bailung, H., and Shukla, P. K.: Observation of ionacoustic shocks in a dusty plasma, Phys. Rev. Lett., 83, 1602 $1605,1999$.

Nemtchinov, I. V., Shuvalov, V. V., Artem'eva, N. A., Ivanov, B. A., Kosarev, I. B., and Trubetskaya, I. A.: Light flashes caused by meteoroids impacts on the lunar surface, Sol. Syst. Res., 32, 99-114, 1998.

Nemtchinov, I. V., Shuvalov, V. V., Artemieva, N. A., Kosarev, I. B., and Trubetskaya, I. A.: Expansion, radiation and condensation of vapor cloud, created by high-velocity impact onto a target in vacuum, Int. J. Impact. Eng., 23, 651-652, 1999.

Nemtchinov, I. V., Shuvalov, V. V., Artemieva, N. A., Kosarev, I. B., and Popel, S. I.: Transient atmosphere generated by large meteoroid impacts onto an atmosphereless cosmic body: gasdynamic and physical processes, Int. J. Impact. Eng., 27, 521-534, 2002.

Newburn Jr., R. L. and Spinard, H.: Spectrometry of 25 comets - Post-Halley updates for 17 comets plus new observations for eight additional comets, Astron. J., 97, 552-569, 1989.

Ostrikov, K.: Reactive plasmas as a versatile nanofabrication tool, Rev. Mod. Phys., 77, 489-511, 2005.

Ostrikov, K. N., Vladimirov, S. V., and Yu, M. Y.: Low-frequency surface waves in a structured magnetized dusty plasma, J. Geophys. Res. Space Phys., 104, 593-599, 1999.

Ostrikov, K. N., Yu, M. Y., and Stenflo, L.: Surface waves in strongly irradiated dusty plasmas, Phys. Rev. E, 61, 782-787, 2000.

Popel, S. I., Vladimirov, S. V., and Tsytovich, V. N.: Theory of modulational interactions in plasmas in the presence of an external magnetic field, Phys. Rep. 259, 327-405, 1995.
Popel, S. I., Yu, M. Y., and Tsytovich, V. N.: Shock waves in plasmas containing variable-charge impurities, Phys. Plasmas, 3, 4313-4315, 1996.

Popel, S. I. and Tsytovich, V. N.: Shocks in Space Dusty Plasmas, Astrophys. Space Sci., 264, 219-226, 1999.

Popel, S. I., Gisko, A. A., Golub', A. P., Losseva, T. V., Bingham, R., and Shukla, P. K.: Shock waves in charge-varying dusty plasmas and the effect of electromagnetic radiation, Phys. Plasmas, 7, 2410-2416, 2000.

Popel, S. I., Golub' A. P., and Losseva, T.V.: Dust ion-acoustic shock-wave structures: theory and laboratory experiments, JETP Lett., 74, 362-366, 2001.

Popel, S. I., Gisko, A. A., Losseva, T. V., and Vladimirov, S. V.: Dust ion acoustic bow shock in interaction of solar wind with cometary coma, 30th EPS Conference on Controlled Fusion and Plasma Physics, St. Petersburg, European Conference Abstracts, 27A, P-4.125, 2003.

Popel, S. I., Losseva, T. V., Merlino, R. L., Andreev, S. N., and Golub', A. P.: Dissipative processes and dust ion-acoustic shocks in a Q machine device, Phys. Plasmas, 12, 054501, 4 p., 2005.

Potter Jr., A. E. and Morgan, T. M.: Discovery of sodium and potassium vapor in the atmosphere of the Moon, Science, 241, 675680, 1988.

Rickman, H.: Composition and physical properties of comets, in: Solar System Ices, Kluwer, Dordrecht, 395-417, 1998.

Smyth, W. H. and Marconi, M. L.: Theoretical overview and modelling of the sodium and potassium atmospheres of the Moon, Astrophys. J., 443, 371-392, 1995.

Singh, P. D., de Almeida, A. A., and Huebner, W. F.: Dust release rates and dust-to-gas mass ratios of eight comets, Astron. J., 104, 848-858, 1992.

Storrs, A. D., Cochran, A. L., and Barker, E. S.: Spectrophotometry of the continuum in 18 comets, Icarus, 98, 163-178, 1992.

Tsytovich, V. N.: Dust plasma crystals, drops, and clouds, PhysicsUspekhi, 40, 53-94, 1997.

Vaisberg, O. L., Smirnov, V., Omelchenko, A., Gorn, L., and Iovlev, M.: Spatial and mass distribution of low-mass dust particles (m less than 10 to the $-10^{\text {th }} \mathrm{g}$ ) in comet P/Halley's coma, Astron. Astrophys., 187, 753-760, 1987.

Verheest, F.: Waves in Dusty Space Plasmas, Kluwer, Dordrecht, 2000.

Vitjazev, A. V., Pechernikova, G. V., and Safronov, V. S.: Terrestrial Planets: Origin and Early Evolution (in Russian), Nauka, Moscow, 1990.

Vladimirov, S. V. and Ostrikov, K.: Dynamic self-organization phenomena in complex ionized gas systems: new paradigms and technological aspects, Phys. Reports, 393, 175-380, 2004.

Zetzer, J. I., Kiselev, Y. N., Poklad, Y. V., and Rybakov, V. A.: Formation of microscale particles in active geophysical experiments "North Star", in: Nano- and Microscale particles in Geophysical Processes, edited by: Adushkin, V. V. and Popel, S. I., The Moscow Inst. of Physics and Technology (in Russian), Moscow, 116-122, 2006. 